\title{
Biosignal and Context Monitoring: Distributed Multimedia Applications of Body Area Networks in Healthcare
}

V.M. Jones, ${ }^{\# 1}$, R. Huis in’t Veld ${ }^{* 2}$, T. Tonis ${ }^{* 3}$, R.B. Bults ${ }^{\# 4}$, B. van Beijnum ${ }^{\# 5}$, I. Widya ${ }^{\# 6}$, M. VollenbroekHutten $^{* 7}$, H. Hermens ${ }^{\# 8}$.

${ }^{\#}$ Faculty of Electrical Engineering, Mathematics and Computer Science, University of Twente

Drienerlolaan 5, 7522 NB Enschede, The Netherlands

${ }^{1}$ V.M.Jones@utwente.nl

R.G.A.bults@utwente.nl

beijnumeewi.utwente.nl

widyacewi.utwente.nl

H.J.Hermens@utwente.nl

"Roessingh Research and Development

Roessinghsbleekweg 33b, 7522 AH Enschede, The Netherlands

R.Huisintvelderrd.nl

T. Toniserrd.nl

M.Vollenbroek@rrd.nl

\begin{abstract}
We are investigating the use of Body Area Networks (BANs), wearable sensors and wireless communications for measuring, processing, transmission, interpretation and display of biosignals. The goal is to provide telemonitoring and teletreatment services for patients. The remote health professional can view a multimedia display which includes graphical and numerical representation of patients' biosignals. Addition of feedback-control enables teletreatment services; teletreatment can be delivered to the patient via multiple modalities including tactile, text, auditory and visual. We describe the health BAN and a generic mobile health service platform and two context aware applications. The epilepsy application illustrates processing and interpretation of multisource, multimedia BAN data. The chronic pain application illustrates multi-modal feedback and treatment, with patients able to view their own biosignals on their handheld device.
\end{abstract}

\section{INTRODUCTION}

A multidisciplinary team of computer scientists, clinicians and biomedical engineers have been researching mobile health systems based on Body Area Networks (BANs) since 2001 [1]. Our definition of a health BAN is a network of communicating devices (sensors, actuators etc.) worn on, around or in the body which provides mobile health services to the user. The patient wears a BAN equipped with biosensors and other devices (eg a positioning device) whose output is processed and transmitted to a remote healthcare professional. The healthcare professional can view a multimedia display including graphical and numerical representation of multiple biosignals and other (synchronised) measurements of the patient and their environment, or selectable subsets of the same (either in real-time or stored). By including a feedback loop and actuation as well as sensing, telemonitoring services can be augmented with feedback and control enabling teletreatment services. Such services, especially when automated or semi-automated, require accurate and reliable processing, transmission and interpretation of the output of multiple biosignal sources in combination with context sources which may include visual, auditory, text and other types of information. In our system we combine output (discrete and continuous) from multiple sensing and context sources and deliver feedback and treatment to the user via multiple modalities including tactile, text, auditory signals and images. In future clinical applications we anticipate direct delivery of treatment by means of actuation of body worn devices such as implanted medication pumps.

The first prototype health BAN was implemented and trialled during the IST project MobiHealth [2]. The core BAN device, the Mobile Base Unit (MBU), acts as a communication gateway to other networks and takes care of local storage and processing. The MBU has been implemented on a number of different PDAs and smart phones (e.g. IPAQ 3870, Qtek 9090, HCT P3600). The m-health service platform and a number of variants of the health BAN, with different sensor sets integrated, were trialled in four European countries, with various biosignals monitored and transmitted to remote healthcare centres over GPRS and UMTS. The nine trials in MobiHealth included telemonitoring for cardiology and COPD (respiratory insufficiency) patients, for pregnant women and for casualties in trauma care. BAN development continued in the Dutch FREEBAND Awareness project [3], the European eTen project HealthService24 [4] and the European eTen project MYOTEL [5]. In the Awareness project we focus on neurology applications (epilepsy, spasticity and chronic pain) and address the issue of adding context awareness, including location awareness, to BAN 
applications. In Awareness we also introduce teletreatment alongside telemonitoring services. In the Myotel project we develop a prototype myofeedback based teletreatment service which enables subjects with neck/shoulder complaints to receive personalised remotely supervised treatment during daily activities. Over the course of these projects we have gained experience of signal processing and interpreting output of a number of sensors and other devices, and different combinations thereof. Sensors which have been integrated into the BAN include electrodes for measuring ECG and EMG, pulse oxymeter, various motion sensors (step counter, 3D accelerometer), temperature and respiration sensors. Other devices which have been incorporated into different variants of the BAN include positioning devices and a multi-modal biofeedback device (vibration and auditory signals).

Fig. 1. shows the elements of the system (in this case, the example is the myofeedback application). In our system, intraBAN communication may be wired or wireless, or a mixture of the two. With front-end supported sensors, communication between the sensors and sensor front-end is wired, and between front-end and MBU wireless (Bluetooth). For non-front-end supported sensors, communication between sensor and MBU is also wireless. The data is transmitted from the MBU to the remote user (eg. GP, physiotherapist or hospital specialist) via a range of wireless networking technologies including WiFi, GPRS and UMTS.

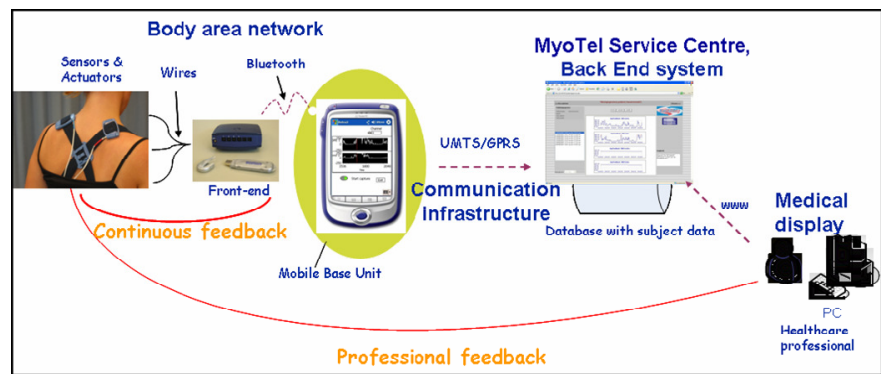

Fig. 1. A BAN-based m-health system

BAN monitoring and treatment services are supported by an infrastructure and distributed service platform known as the m-health service platform (MSP). The MSP incorporates a Jini server whereby BANs and BAN devices are managed and where BAN data is stored and can be accessed by remote users. The MSP is based on the Jini Surrogate Architecture. BAN data (stored data or streamed real-time) can be accessed and displayed by the health professional via a web portal. The professional user can also send device management commands to the BAN as well as treatment and advice back to the patient via the BAN using different modalities.

Section II below describes an application involving multisource, multimedia processing and interpretation of BAN data. Section III shows an application involving multi-modal feedback and treatment. Some of the research challenges arising and planned future work are discussed in Section IV.

\section{MulTi-SOURCE PROCESSING: A CONTEXT AWARE APPLICATION FOR EPILEPSY MONITORING}

This section describes an application which processes and analyses biosignals and context information, including positioning of patient and carers, to identify medical emergencies and facilitate response. The nearest available carer is guided to the patient using GPS positioning and a map display.

\section{A. The Epilepsy Application}

Epilepsy is a serious chronic neurological condition characterized by recurrent unprovoked seizures. The seizures may be related to a brain injury or a family tendency, but in many cases the cause is unknown. The possibility of seizure at any time impacts the patient's life, for example by limiting freedom and bringing feelings of insecurity during daily life. Various medications can be used to prevent seizures, however medication does not guarantee a completely seizure-free life.

Although seizures cannot be reliably prevented, the quality of life of epilepsy patients can be improved. In the Awareness project we developed a detection algorithm and a body worn system (BAN) which warns the epileptic patient of the onset, or imminent onset, of a seizure. Even a small advance warning can be useful, for example in preventing injury. The patient can sit down, or move away from dangerous areas like a busy street or stairs, or put down sharp objects that could cause injury. The detection algorithm analyses heart rate (derived from ECG) in the context of physical activity level of the patient, sensed by a motion sensor, in order to distinguish between heart rate increases caused by physical activity or emotive state and those caused by a seizure. When seizure is detected, as well as warning the patient, the application sends a notification to a remote healthcare location and/or to a voluntary carer. Other kinds of context information used are location and status information. The system determines the locations of the patient and the professionals, also the locations and status (availability) of the informal caregivers. If help from an informal caregiver will be sufficient according to analysis of biosignals and contextual information, the nearest available informal caregiver is selected and notified, and a route from their current location to the patient is presented on a map on their handheld device. Otherwise medical assistance is dispatched in a similar fashion.

Fig. 2. shows the display of biosignals on the portal, which can be viewed by authorised health professionals. Here three traces are displayed against a time axis: ECG, activity level and heart rate (derived from ECG). At the right hand side numeric readouts for activity and heart rate are displayed. 

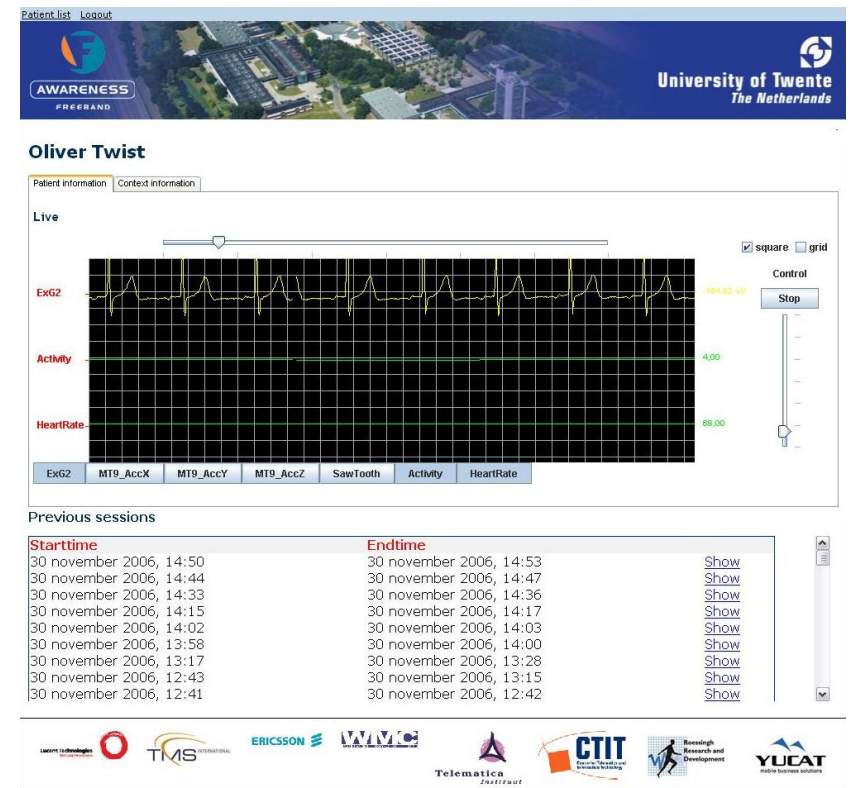

Fig. 2. The m-health portal displaying three biosignal traces: ECG, activity and heart rate

The professional can also view location information. Fig. 3 . shows the map display on the portal, giving the position of the patient and the positions and status (available or not) of all informal carers registered for that patient..

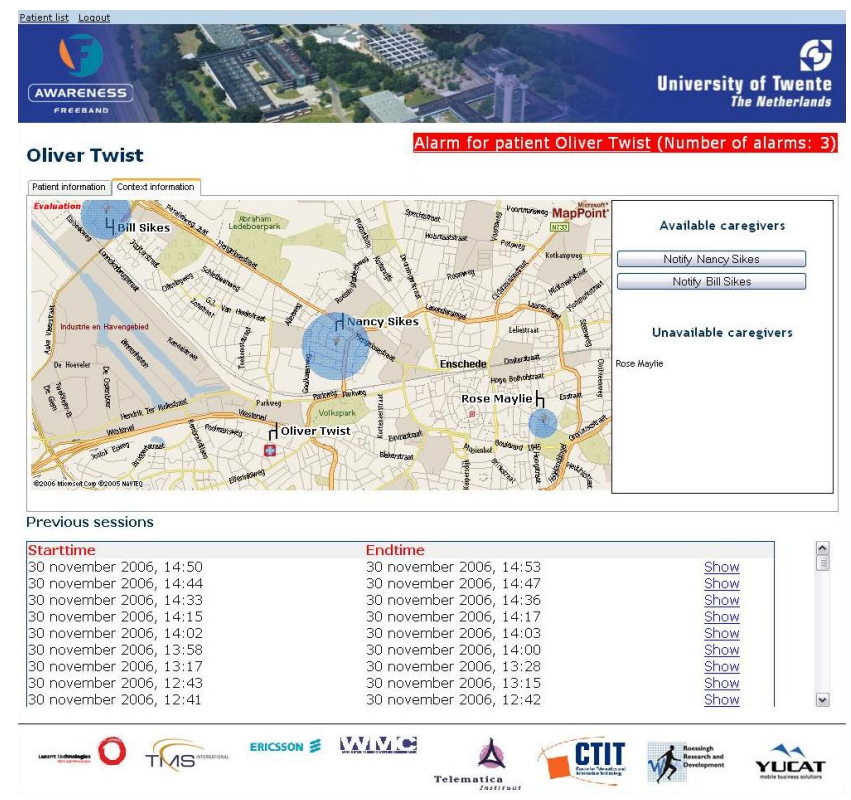

Fig. 3. Epilepsy application: map display

The system also adapts to technical context, namely: prevailing network conditions. If available bandwidth is low, an analysis algorithm runs locally on the BAN and only alarms are sent to the health professional. However, if sufficient bandwidth is available, the biosignals are transmitted to the back-end for processing by a more sophisticated detection algorithm. This also allows the health professional to view a better quality visualization on a PC than can be presented on an MBU.

\section{B. The Epilepsy BAN}

The Epilepsy BAN incorporates an Xsens MT9-B inertial sensor sensing 3D acceleration, and three ECG electrodes ( $\mathrm{Ag} / \mathrm{AgCl}$ contact electrodes) measuring ECG in addition to the MBU (implemented here on an HTC P3600 "PDA phone") and the Mobi8-MT9 sensor front-end from TMSi. The HTC P3600 has an internal GPS device. The electrodes are placed on the patient one centimetre below the right extremitas sternalis, on the fourth left rib below the armpit and at the spinal cord at C7 (reference electrode). Fig. 4. show the components of the epilepsy BAN.

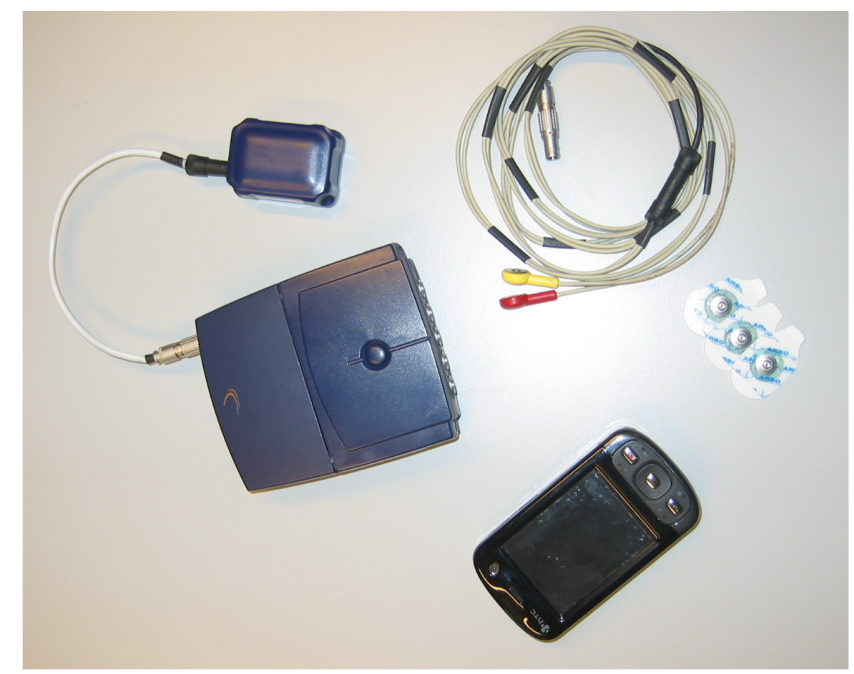

Fig. 4. Epilepsy BAN: electrodes and activity sensor

If a seizure is detected, information from the GPS device together with cell-ID information is used to determine the location of the patient so that appropriate assistance can be sent, depending on the severity of the seizure. The patient receives a notification that a seizure might be imminent. The health professional can view the locations of patient and carers via a GIS on the portal. The nearest carer is dispatched and they can view the patient's location on a map display on their mobile device.

\section{Signal processing and interpretation in the Epilepsy Application}

In patients suffering from a seizure originating in the temporal lobe of the brain there is a change in heart rate prior to, or at onset of, the seizure [6]. Our seizure detection algorithm is based on this change in heart rate and uses in addition posture and activity level information to discriminate between heart rate changes due to epileptic seizure and those due to other causes including physical activity.

Heart rate is derived from the ECG signal, sampled at 1024 $\mathrm{Hz}$, by RTop detection (algorithm adopted from [7]). This beat to beat heart rate is converted to heart rate change by calculating the difference between the mean heart rate in two moving time windows of 10 and 120 seconds.

The inertial data, 3D acceleration data, is sampled at 128 Hz. Activity level [8] and posture (lying or not, detected by 
reference to the earth's gravitational field) are calculated every 10 seconds.

When the patient has a zero or very low activity level and his/her heart rate change rises above a predefined threshold, the heart rate increase is labelled as a possible seizure. If the posture is lying, or changes to lying, the probability that the patient is having a seizure is revised upwards.

\section{Multimodal FeEdBACK: MYOFEEDBACK MONITORING AND TREATMENT}

In this chronic pain application electrodes are used to measure EMG, and from the processed EMG signals a measure called relative rest time (RRT) is derived. When patients have too low a value for RRT, local feedback is given to the patient via three modalities: visualization on the PDA, audio signal and vibration.

\section{The Myofeedback Application}

Neck-shoulder pain is a major problem in western industrialized countries, with significant economic impact since it mainly affects the working population. One of the possible underlying mechanisms for the occurrence of nonspecific neck-shoulder pain (e.g. whiplash, computer-work related) is explained by the so-called Cinderella hypothesis [9]. This hypothesis states that patients with neck-shoulder pain have an insufficient proportion of muscle relaxation time. This hypothesis provided the motivation for a new variant of the BAN, which can warn patients when muscle relaxation time is insufficient. The myofeedback BAN is currently being developed and trialled in the Myotel project.

This application arose out of previous studies where subjects received local feedback from a standalone system. The pain patient wore a garment under their clothes which incorporated dry surface electrodes. These electrodes continuously measured surface ElectroMyoGraphy (sEMG) of the neck-shoulder, specifically of the trapezius muscle. A local device derived relative rest time (RRT) from the raw sEMG signals. When RRT was below a certain threshold, this device provides local auditory and vibratory feedback to the patient. This biofeedback device was used in the rehabilitation clinic for the treatment of non-specific neck-shoulder pain patients. Subjects wore the system for four weeks during daily activities. At least once a week the therapist visited (or was visited by) the subject to discuss progress. However, during this visit the therapist had to interpret the data immediately and discuss it with the subject. The results showed that continuous feedback during everyday activities enabled quick adaptation of a subject's behaviour and showed long lasting treatment effects [10]. However the need for face-to-face visits, with no prior opportunity for the therapist to examine the data, clearly limited the effectiveness of the service. The consultations normally take about thirty minutes but there is extra overhead due to travel time and time needed for downloading the data. Moreover the need for face-to-face consultation limits the geographical area in which subjects can be treated. Therefore, the system was redeveloped in order to support remote monitoring. The new system transmits sEMG data wirelessly from the Myotel patient BAN to the Jini server, via the m-health service platform. Authorized professionals can access the data from there via the m-health portal (either stored historical data or real time) thus enabling teleconsultation.

The prototype system for remotely supervised myofeedback treatment was further tested in the Netherlands on 10 patients with work related neck shoulder problems [11]. Interestingly, the results of this study showed that the teleservice was slightly more effective on the outcome parameters pain and disability compared to the non-remote treatment. This might partly be explained by the fact that, with the BAN, myofeedback treatment subjects can view their muscle activation and muscle relaxation data on the visual display of the PDA. So, as well as tactile (vibrating) and audio feedback from the biofeedback device, the patients receive visual feedback. Visual feedback is considered to be dominant over tactile feedback and therefore, possibly, more effective. In addition, the vibrating signal that is fed back when muscle relaxation is insufficient in non-remote myofeedback gives a rather negative reinforcement (e.g. "you did something wrong"). In remote myofeedback treatment, the visual feedback on the PDA can provide patients with more positive feedback (e.g. "I see I'm doing quite well"). Finally, while the vibrating signal provides the patient with knowledge of results (e.g. "good or bad"), the visual feedback provides patients with knowledge of performance. The literature indicates that knowledge of performance is associated with better learning curves [12].

\section{E. The Myofeedback BAN}

Fig. 5. shows the Myotel myofeedback BAN.

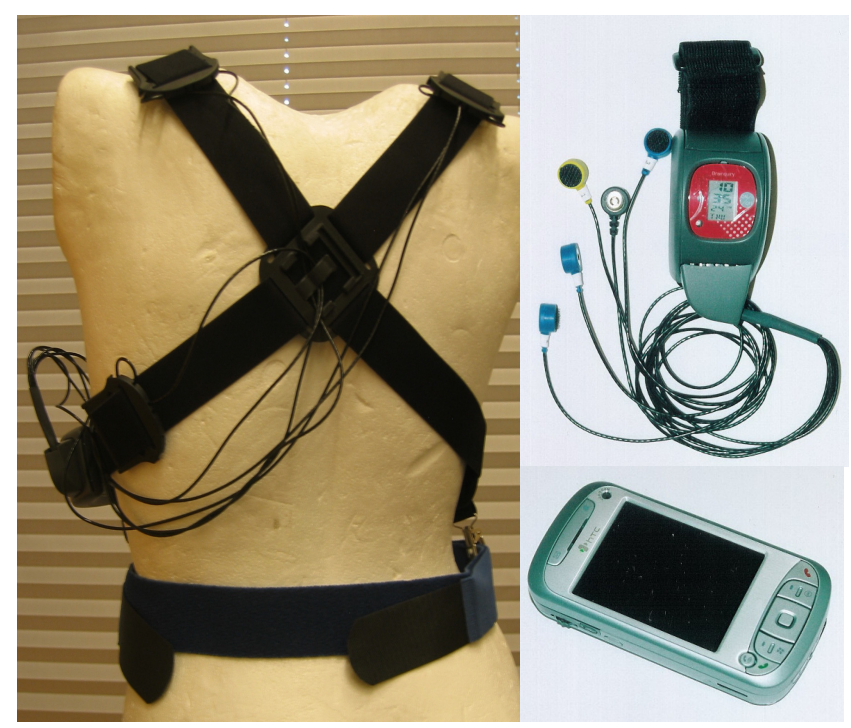

Fig. 5. Myofeedback BAN: garment, electrodes, ReTra and MBU

The Myotel BAN incorporates an MBU implemented on an HTC P3600 and the ReTra system from Brainquiry, used to measure EMG and to give direct feedback. The ReTra incorporates five electrodes (four for EMG measurement and one reference) and is mounted on a harness which is designed 
so that the 4 ECG sensors can be placed over the trapezius muscle (two right and two left). The ReTra system runs algorithms for pre-processing EMG data and can deliver direct feedback to the patient in the form of vibration or auditory signal, indicating that the patient needs to rest. The ReTra also transmits the EMG data to the MBU over a wireless (Bluetooth) link and from the MBU the data is transmitted over one of the available networks (UMTS, GPRS) to the secured server of the m-health service platform.

\section{F. Signal processing and interpretation in the Myofeedback application}

The EMG data is sampled by the ReTra at a rate of $512 \mathrm{~Hz}$ (24 bit ADC) and band pass filtered between 30 and $250 \mathrm{~Hz}$. With this filter setting the effects of movement artefacts in the calculation of Relative muscle Rest Time (RRT) is avoided. RRT is calculated as the time in which the Root Mean Square (RMS) is insufficient, i.e. too low. When RRT is insufficient, vibratory feedback is provided every 10 seconds by the device. In addition, continuous live data-streaming of both the RMS and RRT values for both left and right shoulder can be viewed on the visual display of the patient's MBU (PDA). In other words, continuous visual feedback is provided. Fig. 6. shows the two visualisation screens on the patient's mobile device. The left hand side of Fig. 6. shows the screen display of the calculated Root Mean Square (RMS), displayed for left and right shoulder. By pressing the button marked RRT the display can be switched to Relative Rest Time. The right hand side is the screen display showing RRT, calculated from RMS. Preliminary studies indicate that patients learn very quickly to "read" the graphs and are able to apply relaxation skills to change the graph in the appropriate direction.
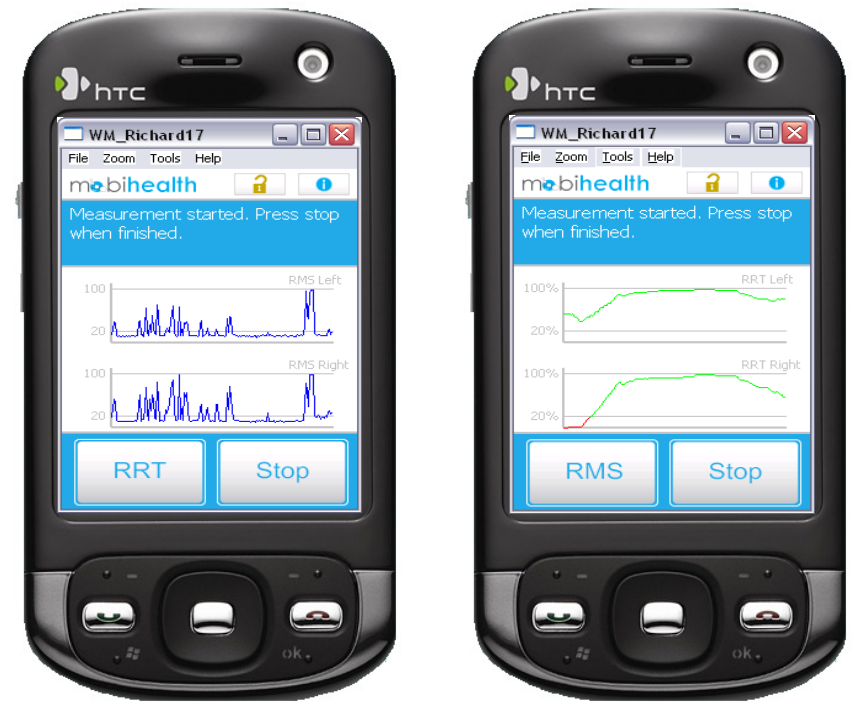

Fig. 6. Visual Myofeedback to patient. Copyright 2008 MobiHealth B.V.

Fig. 7. shows RRT and RMS as displayed on the portal.for viewing by the treating remote health professional..

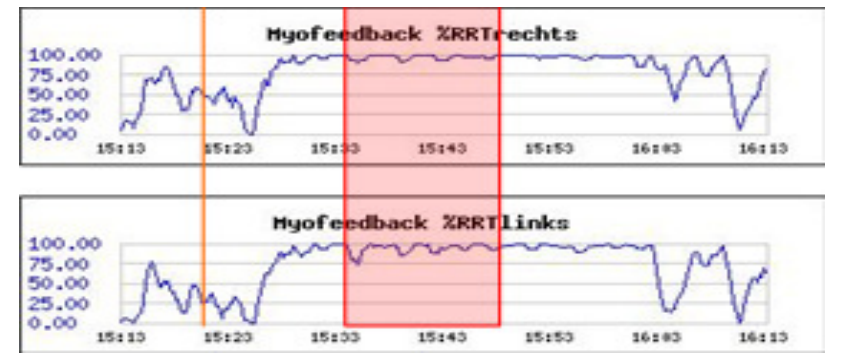

Fig. 7. Myofeedback visualisation for health professional (Relative Rest Time right and left)

Fig. 8. illustrates the overall view of multimodal feedback to the patient. In the micro loop, within the BAN, the patient receives local feedback by auditory, tactile and visual modalities. In the macro loop, the patient can also receive feedback from the professional during remotely supervised training or assessment.

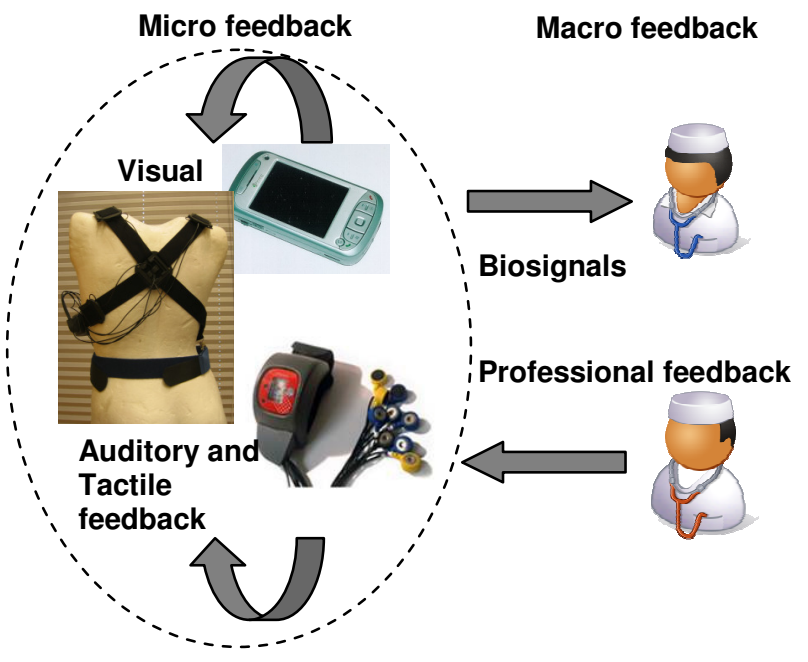

Fig. 8. Multimodal feedback

\section{IV.DISCUSSION AND CONCLUSIONS}

\section{G. Multimedia extensions}

A number of multimedia extensions are envisaged which are expected to enhance the functionality and usability of BAN services.

Video of the patient's posture and movements could be displayed in synchrony with EMG visualisations. This could assist therapists with clinical interpretation and treatment decisions when conducting remote assessment of patients with chronic pain (either in real time or retrospectively). Further, coupling analysis of patients' e-diaries with EMG interpretation could give added context to aid in assessment.

Other modes of feedback to patients could be useful extensions. Adding video feedback for patients could in some cases be more effective than text or auditory feedback alone. For instance, in remotely supervised treatment and training, video showing how relaxation exercises should be done have the potential to improve treatment effectiveness.

Audio-video could also be of value, in the epilepsy scenario for example, to instruct passers-by how to manage an epileptic patient during or following a seizure. 
In another application we anticipate a future scenario involving transmission and fusion of biosignals, audio-video, geo-spatial information and other context sources from the scene of a disaster to emergency teams at the receiving hospitals. This pervasive application is designed to enable telepresence of remote medical expertise by means of augmented reality to aid in first response to major incidents [13], [14].

\section{H. Research Challenges}

There are many technical and research challenges associated with processing, fusion and analysis of biosignals together with other patient related information, especially where mobile and wireless technologies are involved. For example, because of the limited processing power of mobile devices, not all signal processing can be performed locally in the BAN..Transmission cost and battery power also affect best choice of where to perform signal processing and other processing tasks in a distributed system incorporating mobile devices. We are currently investigating dynamic redistribution of signal processing and interpretation tasks to adapt to resource limitations of mobile devices and the changing (wireless) communications environment[16].

Currently the main practical obstacle to success of BAN services is the very limited battery life of the (multiple) mobile devices. For new generations of miniature devices, on which the possibility of ambient intelligence is predicated, various forms of ambient powering seem to offer a solution, although for current medical sensors this solution seems some way away.

Security, safety, correctness and quality of service are critical to acceptance and adoption. An analysis of some of the main technical and socio-technical challenges for m-health technology is given in [15].

\section{Future vision}

With current technology it not very convenient for patients to wear BANs for long periods. In the near future, wearables and implants may alleviate this problem. Further in the future emerging technologies such as bio-nano-technologies may open the way for less obtrusive and more transparent systems and services. In the far future we envision increasing miniaturization enabling the "disappearing BAN" or AmIBAN, incorporating micro- and nano-scale devices, processes and materials, possibly implanted, communicating with the Ambient Intelligent Environment to provide cost-effective, unobtrusive, pervasive, context aware health services [17]. More than ever therefore we will need new methodologies for verifying correctness and safety properties of such highly distributed and autonomous systems [18].

\section{ACKNOWLEDGMENT}

This work is part of the Freeband AWARENESS project (http://awareness.freeband.nl). Freeband is sponsored by the Dutch government under contract BSIK 03025. The Myotel project is supported by the European Union in the framework of the eTen Programme.

\section{REFERENCES}

[1] V. M. Jones, R. G. A. Bults, D. M. Konstantas, and P. A. M. Vierhout, "Healthcare PANs: Personal Area Networks for trauma care and home care," presented at Fourth International Symposium on Wireless Personal Multimedia Communications (WPMC), Aalborg, Denmark, 2001.

[2] MobiHealth, project: http://www.mobihealth.org/.

[3] Freeband AWARENESS http://www.freeband.nl/project.cfm?id=494\&language=en.

[4] eTen HealthService 24 project, http://www.healthservice24.com.

[5] MYOTEL project: http://www.myotel.eu/

[6] Leutmezer, F., Schernthaner, C., Lurger, S., et al, Electrocardiographic changes at the onset of epileptic seizures, Epilepsia, 2003, vol 44 (3), pp 348-354

[7] Christov, I, Real time electrocardiograrm QRS detection using combined adaptive threshold, Biomedical Engineering Online, 2004, $3: 28$

[8] C.V. Bouten, W.P. Verboeket-van de Venne, et al, "Daily physical activity assessment: comparison between movement registration and doubly labeled water", J. Appl. Physiol, 1996, 81, 1019-1026.

[9] Hägg GM. Static workloads and occupational myalgia: A new explanation model. In: Anderson P, Hobart D, Danoff J, eds. Electromyographical Kinesiology, Amsterdam, Elsevier Science Publishers 1991;141-3

[10] Hermens and Hutten, Muscle activation in chronic pain: its treatment using a new approach of myofeedbback. Int J Indus Ergon 2002; 30: 325-36

[11] Huis in 't Veld, Huijgen, Schaake, Hermens, Vollenbroek-Hutten, A staged approach evaluation of remotely supervised myofeedback treatment in women with neck-shoulder pain related to computerwork, Telemedicine and eHealth, 2008 in press.

[12] Schmidt and Lee, Motor control and learning: A behavioral emphasis, San Francisco, United States of America: Human Kinetics, 1999.

[13] Jones, V., and Saranummi, N., MOSAIC vision and scenarios for mobile collaborative work related to health and wellbeing. ICE 2005, 11th International Conference on Concurrent Enterprising, University BW Munich, Germany, 20-22 June 2005, AMI@Work Forum Day: Towards Ambient Intelligence at Work, Vision 2010. Proceedings of the 1st AMI@work communities Forum Day 2005, 'Towards Ambient Intelligence at Work 2010', Munich, Germany, 22 June 2005, Marc Pallot \& Kulwant S Pawar eds.), ISBN 139780853582250.

[14] Jones, V. Karagiannis, G., Heemstra de Groot, S., Ad hoc networking and ambient intelligence to support future disaster response, Proc. ASWN 2005, 5th Workshop on Applications and Services in Wireless Networks, June 29 - July 1st, 2005, Paris, pp. 137-146, Hossam Afifi and Djamal Zeghlache (eds.), Institut National de Telecommunications, Groups des Ecoles des Telecommunications 9, rue Charles Fourier, 91011 Evry Cedex, France, (c) 2005 IEEE. ISBN 2-9156-18-08-9.

[15] Val Jones, F. Incardona, C. Tristram, S. Virtuoso, A. Lymberis (2006). Future Challenges and Recommendations, IN M-Health: Emerging Mobile Health Systems, Robert H. Istepanian, Swamy Laxminarayan, Constantinos S. Pattichis, Editors, Springer. pp.267-72. 624 p. ISBN: 0-387-26558-9.

[16] V.M. Jones, H. Mei, T. Broens, I. Widya, and J. Peuscher, Context Aware Body Area Networks for Telemedicine, Proceedings PacificRim Conference on Multimedia (PCM) 2007, City University of Hong Kong, Hong Kong, 11-14 December 2007.

[17] Val Jones (2006), From BAN to AmI-BAN: micro and nano technologies in future Body Area Networks. IN Val Jones (Ed.) (2006), Proceedings Symposium on Integrated Micro Nano Systems: Convergence of bio and nanotechnologies. Tuesday 20 June, 2006, University of Twente. CTIT Workshop Proceedings Series WP06-03, Enschede, the Netherlands, October 2006, ISSN 1574-0846, ISBN 90365-2449-0.

[18] Val Jones, Aart van Halteren, Dimitri Konstantas, Ing Widya, Richard Bults. An application of augmented MDA for the extended healthcare enterprise. International Journal of Business Process Integration and Management (IJBPIM), ISSN (Print): 1741-8763 ISSN (Online): 17418771, Inderscience Publishers. 